\title{
Study of Silicon Nitride Waveguide Platform for On- Chip Virus Detection: Prospects for COVID-19
}

Raghi S. El Shamy ( $\nabla$ elshamyr@mcmaster.ca )

McMaster University

Mohamed A. Swillam

The American University in Cairo

Xun Li

McMaster University

\section{Research Article}

Keywords: Virus detection, integrated optical biosensors, Si3N4 waveguides

Posted Date: September 8th, 2021

DOI: https://doi.org/10.21203/rs.3.rs-877642/v1

License: (1) This work is licensed under a Creative Commons Attribution 4.0 International License.

Read Full License 


\section{Abstract}

This work presents a rigorous sensitivity analysis of silicon nitride on silicon dioxide strip waveguide for virus detection, focusing on COVID-19. In general, by functionalizing the waveguide surface with specific antibodies layer, we make the optical sensor sensitive only to a particular virus. Unlike conventional virus detection methods such as polymerase chain reaction (PCR), integrated refractive index (RI) optical sensors offer cheap and mass-scale fabrication of compact devices for fast and straightforward detection with high sensitivity and selectivity. Our analysis includes a wide range of wavelengths from visible to mid-infrared. We determined the strip waveguide's single-mode dimensions and the optimum dimensions that maximize the sensitivity to the virus layer attached to its surface at each wavelength. We also compared the strip waveguide to the widely used slot waveguide. Our study shows that silicon nitride strip waveguide working at lower wavelengths is the optimum choice for virus detection as it maximizes both the waveguide sensitivity $\left(\mathrm{S}_{\mathrm{wg}}\right)$ and the figure of merit $(\mathrm{FOM})$ of the sensor. Furthermore, the optimized waveguide can work for a range of viruses. Balanced Mach-Zehnder interferometer (MZI) sensors were designed at different wavelengths showing high FOM at $\lambda=450 \mathrm{~nm}$ ranging from $500 \mathrm{RIU}^{-1}$ up to $1231 \mathrm{RIU}^{-1}$ with $\mathrm{L}_{\mathrm{MZI}}=500 \mu \mathrm{m}$. Different MZI configurations were also studied and compared. Finally, edge coupling from the fiber to the sensor was designed, showing insertion loss (IL) at $\lambda=450 \mathrm{~nm}$ of 4.1 $\mathrm{dB}$ for the design with FOM $=500 \mathrm{RIU}^{-1}$. The obtained coupling efficiencies are higher than recently proposed fiber couplers.

\section{Introduction}

CORONA virus disease of 2019 (COVID-19) pandemic is currently an exceptional threat to human lives all over the world. Its expeditious spread has led to millions of cases and hundreds of thousands of deaths in a few months. Almost all countries worldwide are forced to lockdown for several months to limit the spread of the virus, leading to devastating social and economic effects. In general, many people across the globe lose their life due to viral infection diseases [1]. Hence, simple, fast, cheap, and accurate detection of viruses are of great importance. Polymerase chain reaction (PCR) is one of the well-known methods used for virus detection and it is the primary method used currently for COVID-19 detection [2]. Although this technique is highly sensitive and accurate, it is expensive, time-consuming and involves complex procedures and sample preparation.

Optical refractive index $(\mathrm{RI})$ sensing is one of the main integrated optical techniques used for biodetection [3-8]. RI sensors offer fast, compact and cheap detection with high sensitivities. However, RI sensors are not selective as they only detect the change in the medium (clad) refractive index which can occur due to different substances. A widely used technique to solve this problem in bio-sensing is surface functionalization [9-11]. In surface functionalization, the surface of the sensing waveguide is coated with specific molecules called binder or capture molecules and immobilized through a certain process. These immobilized molecules selectively capture the analyte molecules to be detected from the whole sample. After sensor exposure to the sample, a washing step is needed to make sure that only the analyte of 
interest is present in the sensors' medium (clad) hence, the detected refractive index change is due to this analyte alone.

RI sensors' performance is determined mainly by the figure of merit (FOM) which is the ratio between the sensor sensitivity (S) and full width half maximum (FWHM) of the output spectrum, FOM=S/FWHM. RI sensors' sensitivity $(S)$ can be divided into device sensitivity $\left(S_{D}\right)$ and waveguide sensitivity $\left(S_{w g}\right)$. Device sensitivity is defined as the ratio between the change in the resonance wavelength and the change in the waveguide mode effective index, $S_{D}=d \lambda_{\text {res }} / d n_{\text {eff. }} S_{D}$ is determined by the optical sensor configuration used and its dimensions such as Mach-Zhender interferometer and its arm's length [3-5] or ring resonators and its ring radius [6-8]. While waveguide sensitivity is defined as the ratio between the change in the mode effective index and the change in the medium index, $S_{w g}=d n_{\text {eff }} / d n_{\text {med }} . S_{w g}$ is determined by the sensing waveguide structure such as strip, rib or slot waveguides and its dimensions. The overall sensitivity of the optical sensor is the product of both parameters, $S=S_{D} \times S_{w g}$. Hence, to maximize any RI sensor performance waveguide sensitivity $\left(S_{w g}\right)$ should be maximized.

Silicon nitride on insulator (SiNOI) waveguide platform, where insulator here is the silicon dioxide, offers numerous advantages for various applications [12-15]. Similar to SOI platform SiNOI is CMOS compatible allowing for mass-scale and low cost fabrication [14-15]. It also allows for monolithic integration with silicon devices and other electronic circuitry [12]. The lower refractive index contrast of the SiNOI waveguide compared to SOI reduce scattering loss due to surface roughness resulting in much lower propagation losses [12-14], while still maintaining device compactness. This lower index contrast also makes SiNOI devices more tolerant to fabrication errors [12-13]. In addition, $\mathrm{Si}_{3} \mathrm{~N}_{4}$ thermo-optical coefficient is one order of magnitude lower than $\mathrm{Si}$ [13], hence $\mathrm{Si}_{3} \mathrm{~N}_{4}$ based devices are less sensitive to temperature fluctuations.

Moreover, SiNOI platform has wider transparency range, from visible to mid-infrared, compared to SOI platform [12-15]. This allows the realization of photonic applications outside the telecom bands, such as integrated optical phased arrays for LIDAR applications [16]. Finally, while silicon have large Kerr effect the two-photon absorption (TPA) prevents efficient nonlinear applications. $\mathrm{Si}_{3} \mathrm{~N}_{4}$ on the other hand, has adequate Kerr nonlinearity and almost zero TPA [14-15]. Thus, SiNOI platform allow for frequency comb as well as supercontinuum generation [17-18], which are essential for high data-rate telecommunications, high-resolution spectroscopy and frequency metrology [19].

In this work, we present a detailed sensitivity analysis of silicon nitride $\left(\mathrm{Si}_{3} \mathrm{~N}_{4}\right)$ on silicon dioxide $\left(\mathrm{SiO}_{2}\right)$ strip waveguide for virus detection. The waveguide surface is assumed to be functionalized by the antibodies of the virus to be detected, using a process similar to that in [10], such that the medium index change is only due to this virus.

A Finite difference eigenmode solver (FDE) [20] is used to determine the waveguide dimensions that maximize the waveguide sensitivity $\left(\mathrm{S}_{\mathrm{wg}}\right)$ to a virus layer attached to its surface. Both fundamental quasi-transverse electric (TE) and quasi-transverse magnetic (TM) mode are studied. Moreover, slot 
waveguide was also analyzed and compared to the strip waveguide. Different operating wavelengths were examined from the visible to the mid-infrared range. We found that $S_{w g}$ and FOM increase at lower wavelengths. This analysis is essential to construct a cheap, mass-scale fabrication of compact and highly sensitive RI optical sensor for fast virus detection. The optimized waveguides can be used in different integrated optical devices such as interferometers and resonators to construct the virus sensor. MZI sensors utilizing the optimized waveguides were designed reaching FOM $=1231 \mathrm{RIU}^{-1}$ at $\lambda=450 \mathrm{~nm}$ with $500 \mu \mathrm{m}$ arms' length. We also designed a MZI sensor with waveguide widths above $1 \mu \mathrm{m}$ that can be easily fabricated in simple and cheap facilities. Finally, fiber edge coupling to the sensors chip was studied and optimized, showing higher coupling efficiencies than recently demonstrated fiber couplers. This analysis is generic for any biosensor that uses surface functionalization for selective sensing. One will only need to adjust the optimum waveguide dimensions according to the molecules' size and refractive index to be detected.

\section{Strip Waveguide Analysis And Discussion}

Figure 1(a) shows the $\mathrm{Si}_{3} \mathrm{~N}_{4}$ strip waveguide proposed for virus detection with width (w) and thickness (h) on $\mathrm{SiO}_{2}$ substrate and water clad. The waveguide surface is functionalized for selective detection such that only the virus of interest will adhere to the surface and form a layer. We model this layer by a thickness $h_{\text {vir }}$ equal to the virus diameter and a refractive index $n_{\text {layer, }}$ as shown in Fig.1(a). For COVID-19 the virus diameter is around $80 \mathrm{~nm}$ [21-22] and hence we use $h_{\text {vir }}=80 \mathrm{~nm}$. The refractive index $n_{\text {layer }}$ is given by (1). The value of $n_{\text {layer }}$ changes between the refractive index of the water $n_{\text {water }}$ and the refractive index of the virus $n_{v i r}$ according to the virus coverage fraction $r$.

$$
n_{\text {layer }}=(1-r) \times n_{\text {water }}+r \times n_{\text {vir }}
$$

In this case, virus binding to the immobilized antibodies on the waveguide surface will change this layer refractive index which will accordingly change the waveguide mode effective index.

Different operating wavelengths are studied from visible range $\lambda=450 \mathrm{~nm}$ (blue) and $\lambda=650 \mathrm{~nm}$ (red), to near-infrared $\lambda=980 \mathrm{~nm}$ and $\lambda=1550 \mathrm{~nm}$, and MIR $\lambda=3600 \mathrm{~nm}$. Material dispersion is considered where silicon nitride, silicon dioxide and water refractive index data along the wavelength are obtained from [2324]. At each operating wavelength, we firstly define the single-mode dismensions by determining the maximum width at different thicknesses using FDE solver [20], as shown in Fig. 1(b-c). Then we calculate the waveguide sensitivity $\left(S_{w g}\right)$ at different waveguide dimensions ( $w$ and $h$ ), for both fundamental quasiTE and fundamental quasi-TM modes, which we will denote as TE and TM for simplicity. Note that, unlike most RI sensors designs, here we calculate the surface waveguide sensitivity, $S_{w g}=d n_{\text {eff }} / d n_{\text {layer, }}$, not the bulk sensitivity, $S_{w g}=d n_{\text {eff }} / d n_{\text {clad }}$, which is more accurate for viral detection. $S_{w g}$ is calculated with $n_{\text {layer }}$ around the water index, which means that the waveguides are optimized to have maximum sensitivity at 
minimum virus coverage ( $r$ close to 0 ). Accordingly, the exact virus refractive index $n_{\text {vir }}$ doesn't affect the obtained results.

Figure 2(a-b) show the waveguide sensitivity for different widths and thicknesses at $\lambda=450 \mathrm{~nm}$. Results show that for each waveguide thickness, there is an optimum width that maximizes the waveguide sensitivity. Such behavior is expected [25]. For large waveguide widths most of the mode field is confined inside the silicon nitride core, resulting in low sensitivity. As the width decreases, the mode becomes less confined and the evanescent field in the cladding increase, increasing the sensitivity. However, for small widths, near cut-off, more field moves to the higher (than clad) refractive index substrate which again decreases the sensitivity [25]. Results also show that as the waveguide thickness increases, the optimum width $\left(w_{\text {opt }}\right)$ decreases and optimum sensitivity increases, which is also expected [25]. Hence, waveguides with a higher aspect ratio (AR), $A R=h / w_{o p t}$, can achieve higher $S_{w g}$ reaching 0.513 for the TE mode with $\mathrm{w}=104 \mathrm{~nm}$ and $\mathrm{h}=300 \mathrm{~nm}(\mathrm{AR}=2.88)$. This behavior is similar for all wavelengths. However, high AR waveguide sensors are more challenging to fabricate and expensive as they need a fine mask and complex lithography system to obtain the small waveguide widths needed.

Moreover, at high AR the waveguide sensitivity is very sensitive to width variations. For example, changing the width by only $20 \mathrm{~nm}$ for the TE mode with $A R=2.88$ will significantly reduce $S_{w g}$ to lower than 0.15 , i.e. 3.4 times reduction. Also, this optimum width $\left(w_{\text {opt }}=104 \mathrm{~nm}\right)$ is close to the mode cut-off width ( $\left.w_{\text {cut-off }}=78 \mathrm{~nm}\right)$ and multimode width $\left(w_{M M}=110 \mathrm{~nm}\right)$. Figure 2(c) shows that for high thicknesses with low optimum widths (high AR) TE mode can achieve higher $S_{w g}$ than TM mode while, for lower thicknesses (higher $\mathrm{w}_{\text {opt }}$ and low AR) TM mode exhibit higher $\mathrm{S}_{\mathrm{wg}}$ than TE mode. This is because the TE/TM modes have field discontinuity at the core edges in the $x / y$ direction; hence, decreasing the width/thickness will increase the evanescent field's amount and, hence, sensitivity. Hence, TM mode is optimum for cheap and easy to fabricate large feature size sensors.

Figure 3 shows the optimum $S_{w g}$ and the optimum width $\left(w_{o p t}\right)$ dependence with the AR (or h) at different wavelengths for the TE and TM mode, respectively. We can see that both the operating wavelength and AR have a significant effect on the obtained $S_{w g}$. In addition, the highest $S_{w g}$ is obtained at the lowest operating wavelength $(\lambda=450 \mathrm{~nm})$ and it decreases monotonically as the wavelength increases. It is important to note that, scaling the waveguide dimensions with the wavelength does not result in the same waveguide sensitivity. This is mainly due to the unchanged layer's thickness that changes its refractive index (representing virus attachment). Results also show that TE mode achieves the highest possible $\mathrm{S}_{\mathrm{wg}}$ at every wavelength.

We have also examined $\mathrm{Si}_{3} \mathrm{~N}_{4}$ slot waveguides (TE mode only). Slot waveguides sensitivity increase as slot width decrease. Here we used a slot width $\left(\mathrm{w}_{\text {slot }}\right)$ of $200 \mathrm{~nm}$ as this is the smallest width that can still allow waveguide functionalization [5], [26]. Figure 4(a) shows the maximum slot waveguide sensitivities at $\lambda=1.55 \mu \mathrm{m}$ and $\lambda=3.6 \mu \mathrm{m}$ for the TE mode. At $\lambda=1.55 \mu \mathrm{m} \mathrm{S}_{\mathrm{wg}}=0.22$ while at $\lambda=3.6 \mu \mathrm{m} \mathrm{S} \mathrm{wg}=0.192$. In order to obtain slot mode in the visible range, the slot width should be less than $200 \mathrm{~nm}$ hence not 
suitable for virus detection (functionalization). Consequently, strip waveguides are more suitable for virus detection as they can achieve higher $\mathrm{S}_{\mathrm{wg}}$ at lower wavelengths leading to much higher FOM. In addition, the functionalization process in tiny a $200 \mathrm{~nm}$ slot is challenging. Moreover, strip waveguide offers a simple sensor design as for example there is no need for a strip to slot mode converter.

We also tested the optimized waveguides for different virus diameters (i.e. different $h_{\text {vir }}$ ) from $60 \mathrm{~nm}$ to $200 \mathrm{~nm}$. Figure 4(b) shows that the waveguide sensitivity increase as the virus size increase. It is important to note that, the optimum waveguide dimensions don't change significantly, from the one obtained for $\mathrm{h}_{\mathrm{vir}}=80 \mathrm{~nm}$, by changing the virus size. Hence, the same waveguide can be used for a range of viruses with different diameters.

While waveguide sensitivity is an important parameter, the RI optical sensors' overall performance is determined by the FOM. In both interferometers and resonators, the sensitivity $(S)$ is proportional to $S_{w g} \times \lambda$. However, the FOM is proportional to $S_{w g} / \lambda$ because the FWHM is proportional to $\lambda^{2}$. Hence, operating the sensor at lower wavelengths will achieve the highest performance as $S_{w g}$ increase and $\lambda$ decrease maximizing the FOM. Figure 5 shows fitted curves of both $S_{w g} \times \lambda$ and $S_{w g} / \lambda$ terms versus wavelength for different AR. It can be seen that FOM increased around 8 times from NIR $(\lambda=1.55 \mu \mathrm{m})$ to visible $(\lambda=450 \mathrm{~nm})$ wavelength for both TE and TM modes while the sensitivity decreased only 1.4 times. Moreover, working in the visible wavelength range have another advantage for biosensing as in this range, the losses due to water absorption are minimized. From more than $200 \mathrm{~dB} / \mathrm{cm}$ mode loss at $\lambda=3.6$ $\mu \mathrm{m}$ to less than $3 \times 10^{-3} \mathrm{~dB} / \mathrm{cm}$ in the visible range.

\section{Sensors Design}

Different MZI sensors have been designed to convert the change in the waveguide's effective index to a sensible quantity for COVID-19 detection. The $\mathrm{Si}_{3} \mathrm{~N}_{4}$ waveguide surface will be functionalized with the virus antibodies. In this case, the COVID virus in the sample will be selectively captured by the waveguide. COVID virus binding will change the refractive index of the $80 \mathrm{~nm}$ layer covering the waveguide core. Accordingly, a wavelength shift $(\Delta \lambda)$ in the transmission spectrum of the MZI will occur, from which the virus concentration can then be determined.

For a MZI device with power evenly devided to its arms the transmission spectrum can be derived to be [27]:

$$
T=\cos ^{2}\left(\frac{\Delta \varphi}{2}\right)
$$

with $\Delta \varphi=\frac{2 \pi}{\lambda}\left(n_{e f f, \text { sens }} L_{\text {sens }}-n_{e f f, r e f} L_{r e f}\right)$ 
where $\Delta \varphi$ the phase difference of the MZI arms; $n_{\text {eff,sens, }} n_{\text {eff,ref }}$ and $L_{\text {sens, }} L_{\text {ref }}$ are the waveguide mode effective index and length of the sensing and reference arms of the MZI sensor, respectively.

From which we can get the peak wavelengths as:

$$
\lambda_{\text {pecak }}=\frac{1}{q}\left(n_{\text {eff, sens }} L_{\text {sens }}-n_{\text {eff }, r e f} L_{\text {ref }}\right)
$$

\section{(3) where q an nteger}

Accordingly, the free spectral range (FSR), full-width half maximum (FWHM), sensitivity (S) and FOM of the MZI sensor can be derived as follows [27]:

$$
\begin{aligned}
& F S R=\frac{\lambda^{2}}{n_{\text {eff }, \text { sens }} L_{\text {sens }}-n_{\text {eff }, \text { ref }} L_{\text {ref }}} \\
& F W H M=\frac{\sqrt{2} F S R}{\pi} \\
& S=\frac{d \lambda_{\text {peak }}}{d n}=\frac{\lambda S_{w g} L_{\text {sens }}}{n_{\text {eff }, \text { sens }} L_{\text {sens }}-n_{\text {eff }, \text { ref }} L_{\text {ref }}} \\
& F O M=\frac{S}{F W H M}=\frac{\pi S_{w g} L_{\text {sens }}}{\sqrt{2} \lambda}
\end{aligned}
$$

FOM is the main performance parameter of any RI sensor as it determines the minimum detectable refractive index change. Table 1 shows the dimensions and FOM of different symmetric MZI (s-MZI) sensors designs, $L_{\text {sens }}=L_{\text {ref }}=L_{M Z l}$, at different wavelengths with $A R \approx 1, L_{M Z I}=500 \mu m$ and ideal y-junction for comparison. The optimized strip waveguides from the previous analysis with the virus layer around the core are used as the sensing arm with width $\mathrm{w}_{\text {sens. }}$. Oxide capped waveguides are used as the reference arm with width $w_{\text {ref. }}$. These results are obtained using a FDE solver to determine $n_{\text {eff }}(\lambda)$ of the sensing and reference waveguides. Next, integrated photonics circuit simulator [28] is used to determine $\mathrm{T}(\lambda)$ at different $\mathrm{n}_{\text {layer }}$ (virus concentration) from which S, FWHM and FOM are then calculated. Results exhibit the same response as Fig. 5 with around 8 times greater FOM at the lower (blue) wavelength. Table 1 also shows FOM at $\lambda=450 \mathrm{~nm}$ for different AR, reaching a maximum of $1231 \mathrm{RIU}^{-1}$ at $A R=2.88$. As mentioned before, at higher waveguide widths, TM mode was used as it can reach higher $\mathrm{S}_{\mathrm{wg}}$ than TE mode, see Fig. 2(c). Note that, MIR range was discarded due to its low $\mathrm{S}_{\mathrm{wg}}$ and high (water absorption) losses.

Table 1. FOM of S-MZI sensors with $L_{M Z I}=500 \mu \mathrm{m}$. *Representing TM mode. 


\begin{tabular}{|c|c|c|c|}
\hline$\lambda(\mathrm{nm})$ & AR & Dimensions (nm) & FOM (RIU-1) \\
\hline \multirow{4}{*}{450} & $0.13^{\star}$ & $\mathrm{w}_{\text {sens }}=550, \mathrm{w}_{\text {ref }}=520$ and $\mathrm{h}=70$ & 501 \\
\hline & $0.37 \star$ & $w_{\text {sens }}=270, w_{\text {ref }}=300$ and $h=100$ & 553 \\
\hline & 1 & $\mathrm{w}_{\text {sens }}=138, \mathrm{w}_{\text {ref }}=145$ and $\mathrm{h}=140$ & 812 \\
\hline & 2.88 & $w_{\text {sens }}=104, w_{\text {ref }}=104$ and $h=300$ & 1231 \\
\hline 650 & 1.1 & $w_{\text {sens }}=203, w_{\text {ref }}=230$ and $h=220$ & 454 \\
\hline 980 & 1.2 & $w_{\text {sens }}=300, w_{\text {ref }}=360$ and $h=360$ & 244 \\
\hline 1550 & 1 & $\mathrm{w}_{\text {sens }}=512, \mathrm{w}_{\text {ref }}=850$ and $\mathrm{h}=500$ & 100 \\
\hline
\end{tabular}

Although small waveguide dimensions in the visible range exhibit high sensing performance, the fabrication of such waveguides require complex and expensive lithography systems like electron beam or deep UV lithography. Hence, we want to determine a sensor's performance with a feature size above $1 \mu \mathrm{m}$, which will allow for easy and cheap fabrication. While lower wavelengths exhibit higher performance, the blue wavelength has almost zero sensitivity for small AR waveguides with $\mathrm{w}_{\mathrm{opt}}>1 \mu \mathrm{m}$. Hence, we choose to compare two designs both with TM mode. The first design is operating at low (red) wavelength $\lambda=650$ $\mathrm{nm}$ exhibiting $S_{\mathrm{wg}}$ of 0.115 , and the second is operating at a higher wavelength at $\lambda=980 \mathrm{~nm}$ but demonstrating slightly higher $S_{w g}$ of 0.13 . Table 2 shows the dimensions and the FOM of both MZI sensor designs. We can see that the first design operating at lower (red) wavelength with $A R=0.05$ has a higher FOM of 158 RIU$^{-1}$ even if it exhibits slightly lower $S_{w g}$.

Table 2. FOM of S- MZI sensors with large feature size using $T M$ mode at $L_{M Z I}=500 \mu m$.

\begin{tabular}{lll}
$\lambda(\mathrm{nm})$ & $\begin{array}{l}\text { Dimensions } \\
(\mathbf{n m})\end{array}$ & $\begin{array}{l}\text { FOM } \\
\left(\mathrm{RIU}^{-1}\right)\end{array}$ \\
\hline 650 & $\mathrm{w}_{\text {sens }}=1500, \mathrm{w}_{\text {ref }}=1000$ and $\mathrm{h}=80$ & 158 \\
\hline 980 & $\mathrm{w}_{\text {sens }}=1500, \mathrm{w}_{\text {ref }}=1100$ and $\mathrm{h}=160$ & 127
\end{tabular}

The minimum detectable index change of the virus layer can be calculated from [29] as $\Delta \mathrm{n}_{\min }=1 / F O M$. These values can then be converted to minimum detectable virus coverage $r_{\min }$ using (1). Table 3 shows $\Delta \mathrm{n}_{\min }$ and $\mathrm{r}_{\min }$ for the MZI sensors designs at the blue wavelength with different AR and the design at red 
wavelength optimized for large dimensions $(A R=0.05)$ with $\mathrm{L}_{\mathrm{MZI}}=500 \mu \mathrm{m}$. Note that lower virus concentrations (coverage r) can be detected by increasing the FOM by increasing the MZI sensor length as given in (7).

It is important to note that, silicon nitride waveguides with film thickness great than $300 \mathrm{~nm}$ suffer large stress, and different techniques are used to overcome this problem [30-32]. However, our analysis shows that thin silicon nitride waveguides, with $\mathrm{h}<300 \mathrm{~nm}$, in the visible range are of better sensing performance. In this case, such stress is reduced and a homogeneous index and thickness can be obtained using lowpressure chemical vapor deposition (LP-CVD) [32].

Table 3. $\Delta n_{\min }$ and $R_{\min }$ of $S-M Z I$ sensors with $L_{M Z I}=500 \mu m$. *Representing TM mode.

\begin{tabular}{llll}
$\lambda(\mathrm{nm})$ & AR & $\Delta \mathrm{n}_{\min }$ & $\mathrm{r}_{\min }(\%)$ \\
\hline \multirow{3}{*}{450} & $0.13^{\star}$ & $2.0 \times 10^{-3}$ & 1.29 \\
\cline { 2 - 4 } & $0.37^{\star}$ & $1.8 \times 10^{-3}$ & 1.16 \\
\hline & 1 & $1.2 \times 10^{-3}$ & 0.79 \\
& 2.88 & $8.1 \times 10^{-4}$ & 0.52 \\
\hline 650 & $0.05^{\star}$ & $6.3 \times 10^{-3}$ & 3.73
\end{tabular}

Finally, different MZI configurations were studied and compared for sensing, namely symmetric MZI (sMZI), asymmetric MZI (a-MZI) and loop terminated MZI (LT-MZI) shown in Fig. 6. The simulated results of the different configurations are summarized in Table 4 for the design of TM mode with $\mathrm{w}_{\text {sens }}=270 \mathrm{~nm}$, $\mathrm{h}=100 \mathrm{~nm}$ and $\mathrm{w}_{\text {ref }}=300 \mathrm{~nm}$ at $\lambda=450 \mathrm{~nm}$ and $\mathrm{L}_{\text {sens }}=500 \mu \mathrm{m}$. While s-MZI ( $\left.L_{\text {sens }}=L_{\text {ref }}\right)$ sensitivity is determined only by its waveguide structures, i.e. $\Delta n_{\text {eff }}=n_{\text {eff,sens }}-n_{\text {eff,ref, }}$ a-MZI $\left(L_{\text {sens }}=L_{\text {ref }}+\Delta L\right)$ sensitivity can be engineered using $\Delta \mathrm{L}=\mathrm{L}_{\text {sens }} \mathrm{L}_{\text {ref, }}$ according to (6). However, both structures will exhibit almost the same FOM for the same $L_{\text {sens. }}$. On the other hand, LT-MZI is a recently proposed design [33] that consists of a conventional MZI with a loop connecting the output directional coupler arms, reflecting back the wave to the interferometer. For the same waveguide structure and $\mathrm{L}_{\text {sens, }}$ LT-MZI will exhibit the same sensitivity with the conventional MZI while the FWHM will reduce to half resulting in twice the FOM. The LT-MZI directional couplers are also assumed to be ideal 3-dB couplers. The asymmetric LT-MZI can also be used to control the sensitivity using $\Delta \mathrm{L}$ as in the a-MZI case.

Table 4. FOM and $S$ of the TM mode for different MZI sensors configurations with $w_{\text {sens }}=270 \mathrm{~nm}, \mathrm{~h}=100$ $\mathrm{nm}$ and $\mathrm{w}_{\text {ref }}=300 \mathrm{~nm}$ at $\lambda=450 \mathrm{~nm}$ and $\mathrm{L}_{\text {sens }}=500 \mu \mathrm{m}$. 


\begin{tabular}{|cccl|}
\hline & & S (nm/RIU) & FOM (RIU-1 $)$ \\
\hline \multirow{2}{*}{ s-MZI } & & 3098 & 553 \\
a-MZI & $\Delta \mathrm{L}=30 \mu \mathrm{m}$ & 1316 & 540 \\
\cline { 2 - 4 } & $\Delta \mathrm{L}=5 \mu \mathrm{m}$ & 5579 & 530 \\
\hline \multirow{2}{*}{ LT-MZI } & & 3098 & 1106 \\
\hline
\end{tabular}

\section{Edge Fiber Coupling}

Recently, many efforts have been done to couple light from fiber to $\mathrm{Si}_{3} \mathrm{~N}_{4}$ platform in the NIR range

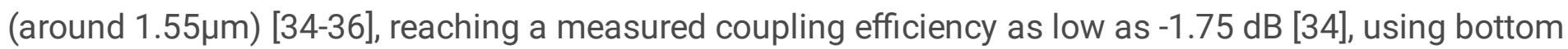
multilayer reflector and apodized grating coupler. However, few work have been pubished for coupling in the visible wavelength range [37-39].

In this section, we study the coupling from single-mode fibers [40] in the visible region (blue and red) to the silicon nitride chip through edge coupling again using an FDE solver. We focus on the coupling to the TM mode sensors designs mentioned in the previous section, which can achieve high coupling efficiencies and high FOM with waveguide widths larger than $250 \mathrm{~nm}$, see Table 1 and Table 2. Design 1: $\mathrm{w}_{\text {sens }}=550 \mathrm{~nm}, \mathrm{~h}=70 \mathrm{~nm}(\mathrm{AR}=0.13)$ and Design 2: $\mathrm{w}_{\text {sens }}=270 \mathrm{~nm}, \mathrm{~h}=100 \mathrm{~nm}(\mathrm{AR}=0.37)$ both at $\lambda=450$ $\mathrm{nm}$. While Design 3: $w_{\text {sens }}=1500 \mathrm{~nm}, \mathrm{~h}=80 \mathrm{~nm}(\mathrm{AR}=0.05)$ at $\lambda=650 \mathrm{~nm}$ for large feature size sensor $(\mathrm{w}>1$ $\mu \mathrm{m})$. Figure 7(a) shows the coupling efficiency at blue and red wavelengths to a waveguide with thickness $h=20 \mathrm{~nm}$ and $\mathrm{h}=40 \mathrm{~nm}$, respectively. A maximum coupling efficiency of $93 \%$ and $92.7 \%$ can be achieved from the fiber to the waveguide TM mode at $\lambda=450 \mathrm{~nm}$ with $\mathrm{w}=600 \mathrm{~nm}$ and at $\lambda=650 \mathrm{~nm}$ with $\mathrm{w}=565 \mathrm{~nm}$, respectively. Note that, waveguides with higher thicknesses exhibit significantly lower coupling for $w>250 \mathrm{~nm}$. Hence, there is a mismatch between the waveguides' dimensions with optimum fiber coupling $\left(w_{c p}, h_{c p l}\right)$ and optimum sensing $\left(w_{\text {sens }}, h_{\text {sens }}\right)$, as shown in Fig. $7(b)$. Accordingly, the coupling between these two waveguides was studied and the insertion loss (IL) was determined for the different sensing waveguides. For each design we optimize the waveguide-waveguide coupling, $w_{c p l} \times h_{c p l} \rightarrow w_{\text {out }} \times h_{\text {sens, }}$ by changing the output waveguide width ( $w_{\text {out }}$ ), which can then be converted to $\mathrm{w}_{\text {sens }}$ with significantly low losses using a taper. Hence, for the blue wavelength the IL from the fiber to the optimum coupling waveguide, $\mathrm{w}_{\mathrm{cpl}}=600 \mathrm{~nm}$ and $\mathrm{h}_{\mathrm{cpl}}=20 \mathrm{~nm}$, is $0.3 \mathrm{~dB}$. The waveguide-waveguide coupling for the sensing waveguide with $h_{\text {sens }}=70 \mathrm{~nm}$ (Design 1) shows IL=3.8 dB with optimum $w_{\text {out }}$ of $2600 \mathrm{~nm}$. While for the sensing waveguide with $h_{\text {sens }}=100 \mathrm{~nm}$ (Design 2) IL=4.8 dB at $w_{\text {out }}=2700 \mathrm{~nm}$. Hence, the fibre's overall coupling loss to the sensing waveguide is $4.1 \mathrm{~dB}$ and $5.1 \mathrm{~dB}$, respectively. For the red wavelength (Design 3), with $\mathrm{w}>1 \mu \mathrm{m}$, the fiber-coupling waveguide IL is $1.37 \mathrm{~dB}$ at $\mathrm{w}_{\mathrm{cpl}}=1030 \mathrm{~nm}$ and $\mathrm{h}_{\mathrm{cpl}}=40 \mathrm{~nm}$. While waveguide-waveguide coupling exhibits $\mathrm{IL}=1.1 \mathrm{~dB}$ for $\mathrm{w}_{\text {out }}=1940 \mathrm{~nm}$ to the sensing waveguide with $h_{\text {sens }}=80 \mathrm{~nm}$, resulting in an overall coupling loss of $2.47 \mathrm{~dB}$. 
These designs exhibit higher coupling efficiencies than most fiber couplers proposed for the $\mathrm{Si}_{3} \mathrm{~N}_{4}$ platform at the same wavelength range [37-39]. This is mainly due to the different waveguide dimensions, as the optimum waveguides for sensing have small core thickness dimensions. Thus, exhibiting large mode size which leads to better matching with the fiber mode. The recently proposed fiber couplers and our proposed ones are summarized in Table 5.

Table 5. Comparison of our proposed fiber couplers and recently demonstrated ones, showing the output waveguide $(w \times h)$ in each case with $(S)$ and $(M)$ denoting simulated and measured results, respectively.

\begin{tabular}{lllll} 
& $\lambda(\mathrm{nm})$ & Waveguide $(\mathrm{nm})$ & Technique & Coupling Loss (dB) \\
\hline [37] & 660 & $700 \times 100$ & Grating & $4.2(\mathrm{M})$ \\
\hline \multirow{3}{*}[38]{} & 532 & $350 \times 180$ & Grating & $6(\mathrm{~S})$ \\
\cline { 2 - 5 } & 640 & $340 \times 220$ & Grating & $6.6(\mathrm{~S})$ \\
& & & & $7.5(\mathrm{M})$ \\
\hline [39] & $430-648$ & $340 \times 135$ & Edge & $8-8.9(\mathrm{M})$ \\
\hline & & $550 \times 70$ & Edge with h step & $4.1(\mathrm{~S})$ \\
\cline { 3 - 5 } & 450 & $270 \times 100$ & Edge with h step & $5.1(\mathrm{~S})$ \\
\cline { 2 - 5 } & 650 & $1500 \times 80$ & Edge with h step & $2.47(\mathrm{~S})$
\end{tabular}

\section{Conclusion}

We propose a $\mathrm{Si}_{3} \mathrm{~N}_{4}$ strip waveguide to be used as the sensing arm in different integrated optical sensors configurations for virus detection. Integrated RI sensors offer fast, cheap and simple detection when compared to the existing methods like the PCR, which is expensive and involves complex procedures. Our study shows that the $\mathrm{Si}_{3} \mathrm{~N}_{4}$ strip waveguide sensors can achieve high sensitivity, and with surface functionalization, it can detect only a specific virus for high selectivity. As an example, in this study, we focus on COVID-19 detection. Our analysis determines the waveguide dimensions that maximize the sensitivity to the virus layer attached to its surface. The optimum dimensions were determined for different wavelengths from the visible to the MIR and for both fundamental quasi-TE and quasi-TM modes. In addition, we compared the silicon nitride strip waveguide to the slot waveguide. Results show that strip waveguide operating at low wavelengths is the best choice for virus detection. MZI sensors were designed offering a FOM as high as $1231 \mathrm{RIU}^{-1}$ for $L_{\mathrm{MZI}}=500 \mu \mathrm{m}$ at $\lambda=450 \mathrm{~nm}$. Finally, edge coupling from the fiber to the waveguide sensor was studied, showing only $4.1 \mathrm{~dB}$ insertion loss at $\lambda=450 \mathrm{~nm}$ for MZI design with FOM=500 RIU $^{-1}$.

\section{Declarations}




\section{Conflict of interest}

The authors declare that they have no conflict of interest.

\section{Author Contributions}

M.A. Swillam conceived the basic idea. R.S. El Shamy validated the concept, designed and optimized the proposed devices through computer-aided simulations and wrote the paper. M.A. Swillam helped in writing and revising the manuscript. M.A. Swillam and X. Li supervised the entire project. All the authors contributed to the general discussion and revision of the manuscript.

\section{References}

1. WHO | The top 10 causes of death. WHO (2017). Available at: http://www.who.int/mediacentre/factsheets/fs310/en/. (Accessed: 9th June 2017).

2. Piatak, M., Saag, M.S., Yang, L.C., Clark, S.J., Kappes, J.C., Luk, K.C., Hahn, B.H., Shaw, G.M. and Lifson, J.D., 1993. High levels of HIV-1 in plasma during all stages of infection determined by competitive PCR. Science, 259(5102), pp.1749-1754.

3. Bastos, A.R., Vicente, C., Oliveira-Silva, R., Silva, N.J., Tacão, M., Costa, J.P.D., Lima, M., André, P.S. and Ferreira, R.A., 2018. Integrated optical Mach-Zehnder interferometer based on organic-inorganic hybrids for photonics-on-a-chip biosensing applications. Sensors, 18(3), p.840.

4. Murib, M.S., Martens, D. and Bienstman, P., 2018. Label-free real-time optical monitoring of DNA hybridization using SiN Mach-Zehnder interferometer-based integrated biosensing platform. Journal of biomedical optics, 23(12), p.127002.

5. Liu, Q., Tu, X., Kim, K.W., Kee, J.S., Shin, Y., Han, K., Yoon, Y.J., Lo, G.Q. and Park, M.K., 2013. Highly sensitive Mach-Zehnder interferometer biosensor based on silicon nitride slot waveguide. Sensors and Actuators B: Chemical, 188, pp.681-688.

6. Bryan, M.R., Steiner, D.J., Cognetti, J.S. and Miller, B.L., 2018, May. Design, manufacture, and testing of a silicon nitride ring resonator-based biosensing platform. In Chemical, Biological, Radiological, Nuclear, and Explosives (CBRNE) Sensing XIX (Vol. 10629, p. 106290Z). International Society for Optics and Photonics.

7. Besselink, G.A., Geuzebroek, D.H., Schreuder, F., Falke, F., Leinse, A. and Heideman, R.G., 2019, March. Microring resonator biosensing platform for sensitive detection of thrombin. In Microfluidics, BioMEMS, and Medical Microsystems XVII (Vol. 10875, p. 1087511). International Society for Optics and Photonics.

8. Al Mahmod, M.J., Hyder, R. and Islam, M.Z., 2018. A highly sensitive metal-insulator-metal ring resonator-based nanophotonic structure for biosensing applications. IEEE Sensors Journal, 18(16), pp.6563-6568. 
9. Angelopoulou, M., Kakabakos, S. and Petrou, P., 2018. Label-Free Biosensors Based onto Monolithically Integrated onto Silicon Optical Transducers. Chemosensors, 6(4), p.52.

10. Sakamoto, H., Minpou, Y., Sawai, T., Enami, Y. and Suye, S.I., 2016. A novel optical biosensing system using Mach-Zehnder-type optical waveguide for influenza virus detection. Applied biochemistry and biotechnology, 178(4), pp.687-694.

11. A. Ymeti et al., "Fast ultrasensitive virus detection using a young interferometer sensor", Nano Lett., vol. 7, pp. 394-397, 2007.

12. Porcel, M.A., Hinojosa, A., Jans, H., Stassen, A., Goyvaerts, J., Geuzebroek, D., Geiselmann, M., Dominguez, C. and Artundo, I., 2019. Silicon nitride photonic integration for visible light applications. Optics \& Laser Technology, 112, pp.299-306.

13. Wilmart, Q., El Dirani, H., Tyler, N., Fowler, D., Malhouitre, S., Garcia, S., Casale, M., Kerdiles, S., Hassan, K., Monat, C. and Letartre, X., 2019. A versatile silicon-silicon nitride photonics platform for enhanced functionalities and applications. Applied Sciences, 9(2), p.255.

14. Baets, R., Subramanian, A.Z., Clemmen, S., Kuyken, B., Bienstman, P., Le Thomas, N., Roelkens, G., Van Thourhout, D., Helin, P. and Severi, S., 2016, March. Silicon Photonics: silicon nitride versus silicon-on-insulator. In Optical Fiber Communication Conference (pp. Th3J-1). Optical Society of America.

15. Sharma, T., Wang, J., Kaushik, B.K., Cheng, Z., Kumar, R., Zhao, W. and Li, X., 2020. Review of Recent Progress on Silicon Nitride-based Photonic Integrated Circuits. IEEE Access.

16. Poulton, Christopher V., et al. "Large-scale silicon nitride nanophotonic phased arrays at infrared and visible wavelengths." Optics letters 42.1 (2017): 21-24.

17. S. Miller et al, On-chip frequency comb generation at visible wavelengths via simultaneous secondand third-order optical nonlinearities, Optics Express, 22(22), p.26517-26525 (2014)

18. H. Zhao et al, Visible-to-near-infrared octave spanning supercontinuum generation in a silicon nitride waveguide, Optics Letters, 40(10), p.2177-2180 (2015).

19. Xuan, Yi, et al. "High-Q silicon nitride microresonators exhibiting low-power frequency comb initiation." Optica 3.11 (2016): 1171-1180.

20. Optical Waveguide Design Software - Lumerical MODE Solutions. Lumerical https://www.lumerical.com/products/mode-solutions/.

21. Tavazzi, G., Pellegrini, C., Maurelli, M., Belliato, M., Sciutti, F., Bottazzi, A., Sepe, P.A., Resasco, T., Camporotondo, R., Bruno, R. and Baldanti, F., 2020. Myocardial localization of coronavirus in COVID19 cardiogenic shock. European journal of heart failure.

22. Sahin, A.R., Erdogan, A., Agaoglu, P.M., Dineri, Y., Cakirci, A.Y., Senel, M.E., Okyay, R.A. and Tasdogan, A.M., 2020. 2019 novel coronavirus (COVID-19) outbreak: a review of the current literature. EJMO, 4(1), pp.1-7. 
23. Philipp, H.R., 1973. Optical properties of silicon nitride. Journal of the Electrochemical Society, 120(2), p.295.

24. Palik, E.D. ed., 1998. Handbook of optical constants of solids (Vol. 3). Academic press.

25. Shi, Y., Ma, K. and Dai, D., 2016. Sensitivity enhancement in Si nanophotonic waveguides used for refractive index sensing. Sensors, 16(3), p.324.

26. Barrios, C.A., Banuls, M.J., Gonzalez-Pedro, V., Gylfason, K.B., Sanchez, B., Griol, A., Maquieira, A., Sohlström, H., Holgado, M. and Casquel, R., 2008. Label-free optical biosensing with slotwaveguides. Optics letters, 33(7), pp.708-710.

27. El Shamy, R. S., Swillam, M. A., \& Khalil, D. A. (2019). Mid infrared integrated MZI gas sensor using suspended silicon waveguide. Journal of Lightwave Technology, 37(17), 4394-4400.

28. PIC Design and Simulation Software - Lumerical INTERCONNECT. Lumerical https://www.lumerical.com/products/interconnect/.

29. Luan, E., Shoman, H., Ratner, D. M., Cheung, K. C., \& Chrostowski, L. (2018). Silicon photonic biosensors using label-free detection. Sensors, 18(10), 3519.

30. Luke, K., Dutt, A., Poitras, C.B. and Lipson, M., 2013. Overcoming $\mathrm{Si}_{3} \mathrm{~N}_{4}$ film stress limitations for high quality factor ring resonators. Optics express, 21(19), pp.22829-22833.

31. Krückel, C.J., Fülöp, A., Klintberg, T., Bengtsson, J. and Andrekson, P.A., 2015. Linear and nonlinear characterization of low-stress high-confinement silicon-rich nitride waveguides. Optics express, 23(20), pp.25827-25837.

32. Subramanian, A.Z., Neutens, P., Dhakal, A., Jansen, R., Claes, T., Rottenberg, X., Peyskens, F., Selvaraja, S., Helin, P., Du Bois, B. and Leyssens, K., 2013. Low-loss singlemode PECVD silicon nitride photonic wire waveguides for 532-900 nm wavelength window fabricated within a CMOS pilot line. IEEE Photonics Journal, 5(6), pp.2202809-2202809.

33. El-Rayany, Mohamed M., Raghi S. El Shamy, and Mohamed A. Swillam. "A compact silicon-oninsulator gas sensor." Silicon Photonics XIV. Vol. 10923. International Society for Optics and Photonics, 2019.

34. Hong, J., Spring, A. M., Qiu, F., \& Yokoyama, S. (2019). A high efficiency silicon nitride waveguide grating coupler with a multilayer bottom reflector. Scientific reports, 9(1), 1-8.

35. Mak, J. C., Sacher, W. D., Ying, H., Luo, X., Lo, P. G. Q., \& Poon, J. K. (2018). Multi-layer silicon nitrideon-silicon polarization-independent grating couplers. Optics express, 26(23), 30623-30633.

36. Chen, Y., Halir, R., Molina-Fernández, Í., Cheben, P., \& He, J. J. (2016). High-efficiency apodizedimaging chip-fiber grating coupler for silicon nitride waveguides. Optics letters, 41(21), 5059-5062.

37. Romero-García, S., Merget, F., Zhong, F., Finkelstein, H., \& Witzens, J. (2013). Silicon nitride CMOScompatible platform for integrated photonics applications at visible wavelengths. Optics express, 21(12), 14036-14046.

38. Song, J. H., Kongnyuy, T. D., Troia, B., Saseendran, S. S., Soussan, P., Jansen, R., \& Rottenberg, X. (2019). Grating devices on a silicon nitride technology platform for visible light applications. OSA 
Continuum, 2(4), 1155-1165.

39. Sacher, W. D., Luo, X., Yang, Y., Chen, F. D., Lordello, T., Mak, J. C. \& Roukes, M. L. (2019). Visible-light silicon nitride waveguide devices and implantable neurophotonic probes on thinned $200 \mathrm{~mm}$ silicon wafers. Optics Express, 27(26), 37400-37418.

40. THORLABS:https://www.thorlabs.com/newgrouppage9.cfm?objectgroup_id=949

\section{Figures}
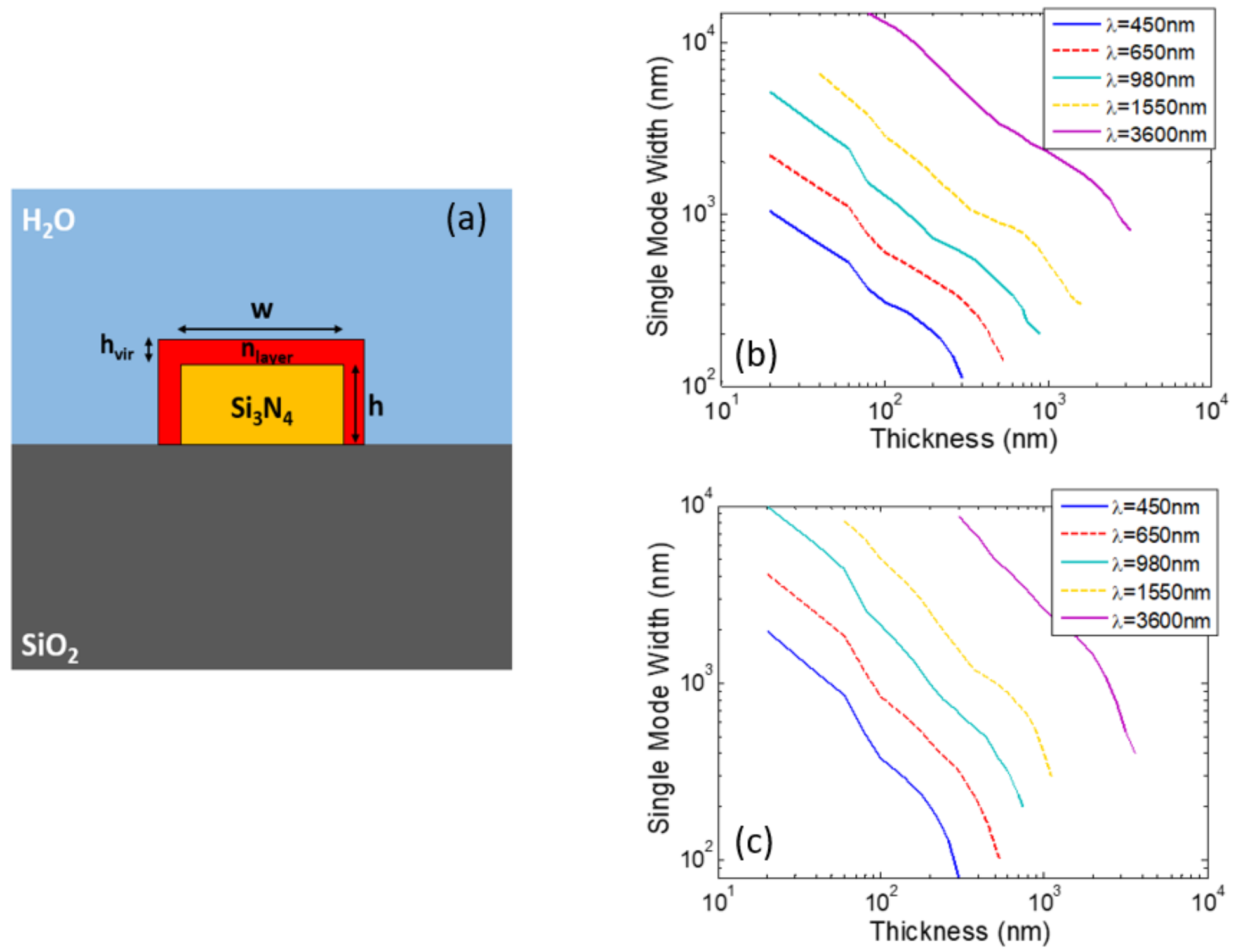

\section{Figure 1}

(a) Schematic of surface functionalized Si3N4 on $\mathrm{SiO} 2$ strip waveguide for virus detection with a layer representing virus attachment. Strip waveguide single-mode width versus waveguide thickness at different wavelengths of: (b) TE mode and (c) TM mode. 

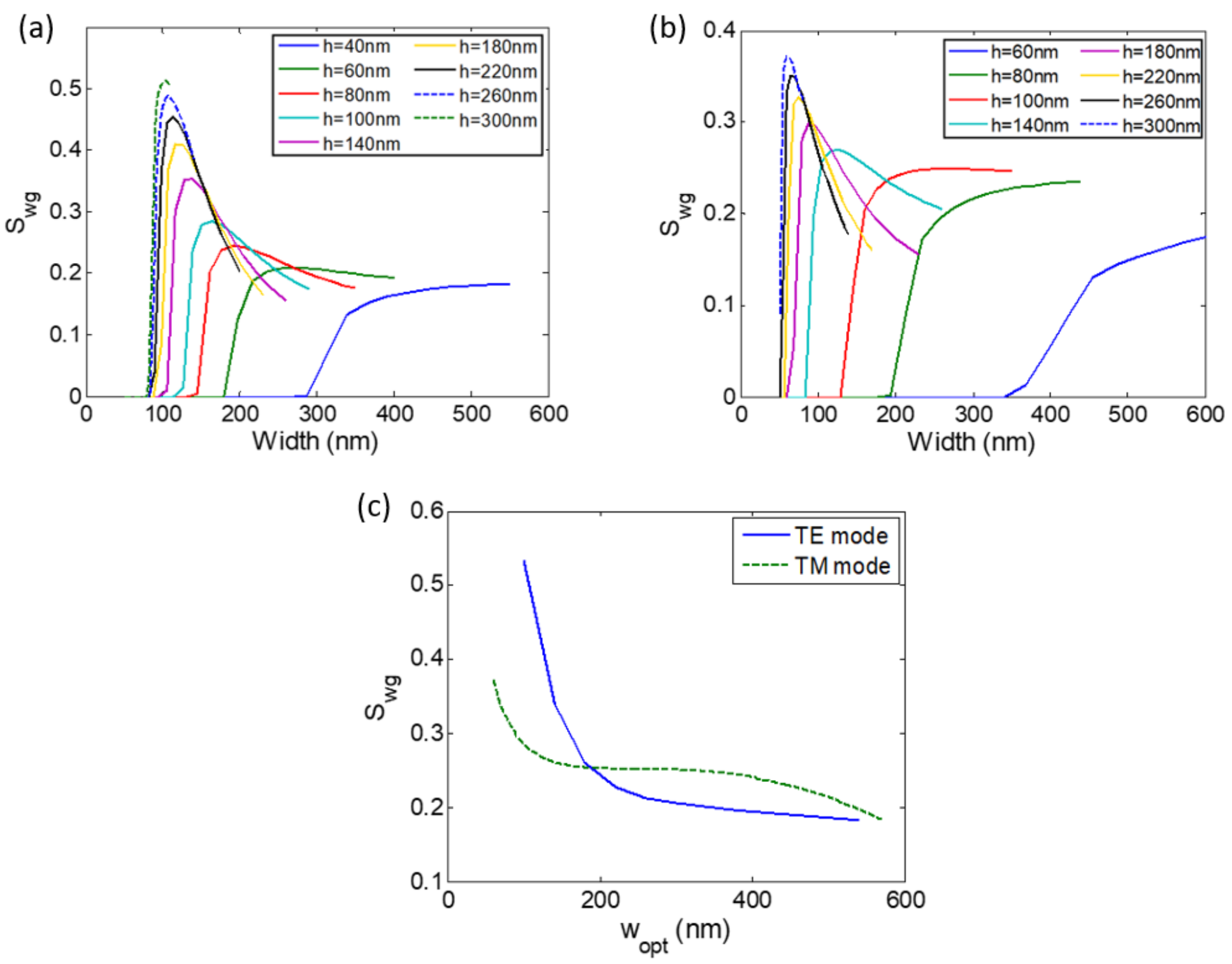

Figure 2

Strip waveguide sensitivity (Swg) versus waveguide width at different thicknesses at $\lambda=450 \mathrm{~nm}$ of: (a) TE mode and (b) TM mode. (c) Strip waveguide sensitivity (Swg) of both TE and TM modes versus the optimum waveguide width (wopt) at $\lambda=450 \mathrm{~nm}$. 
(a)
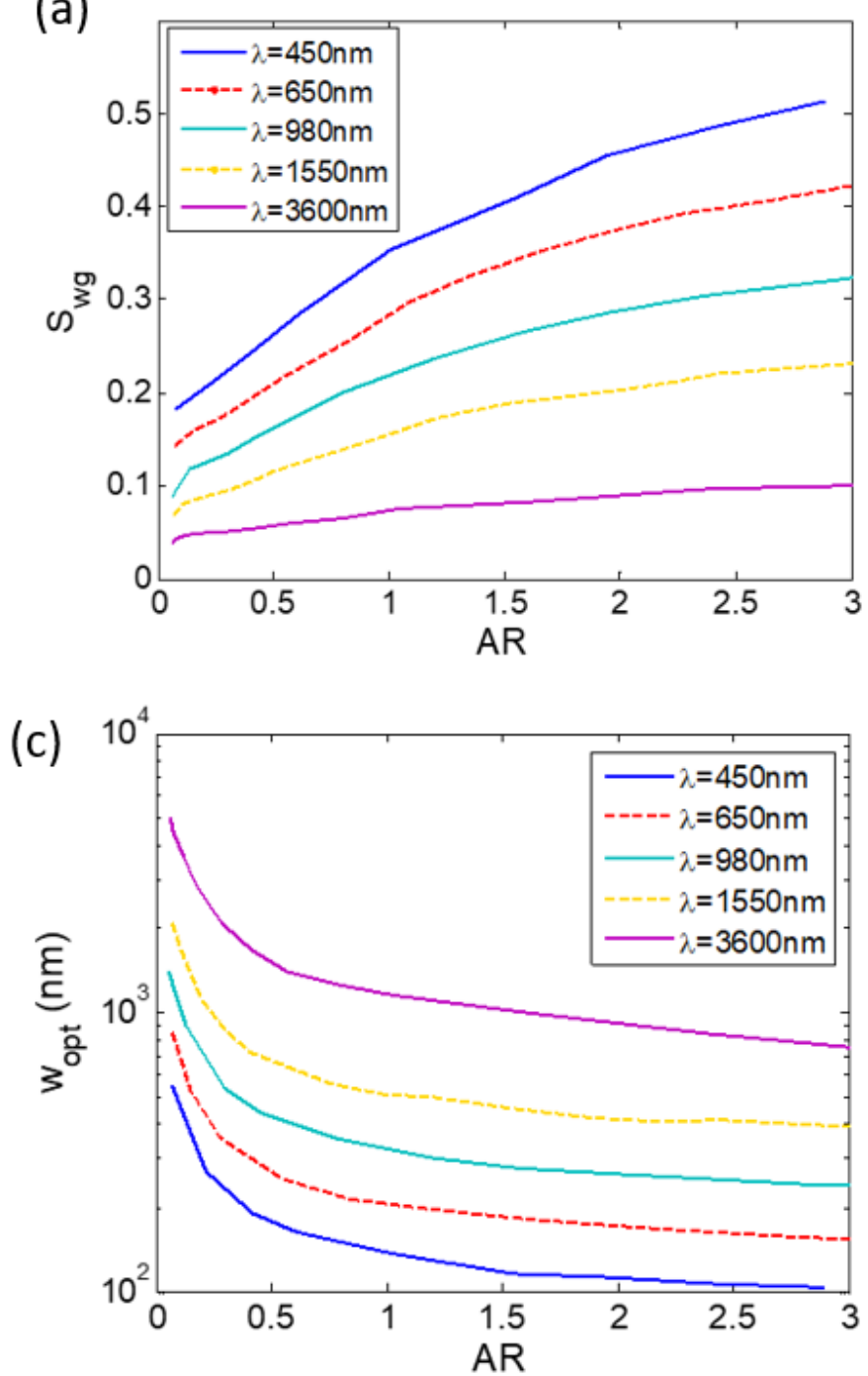
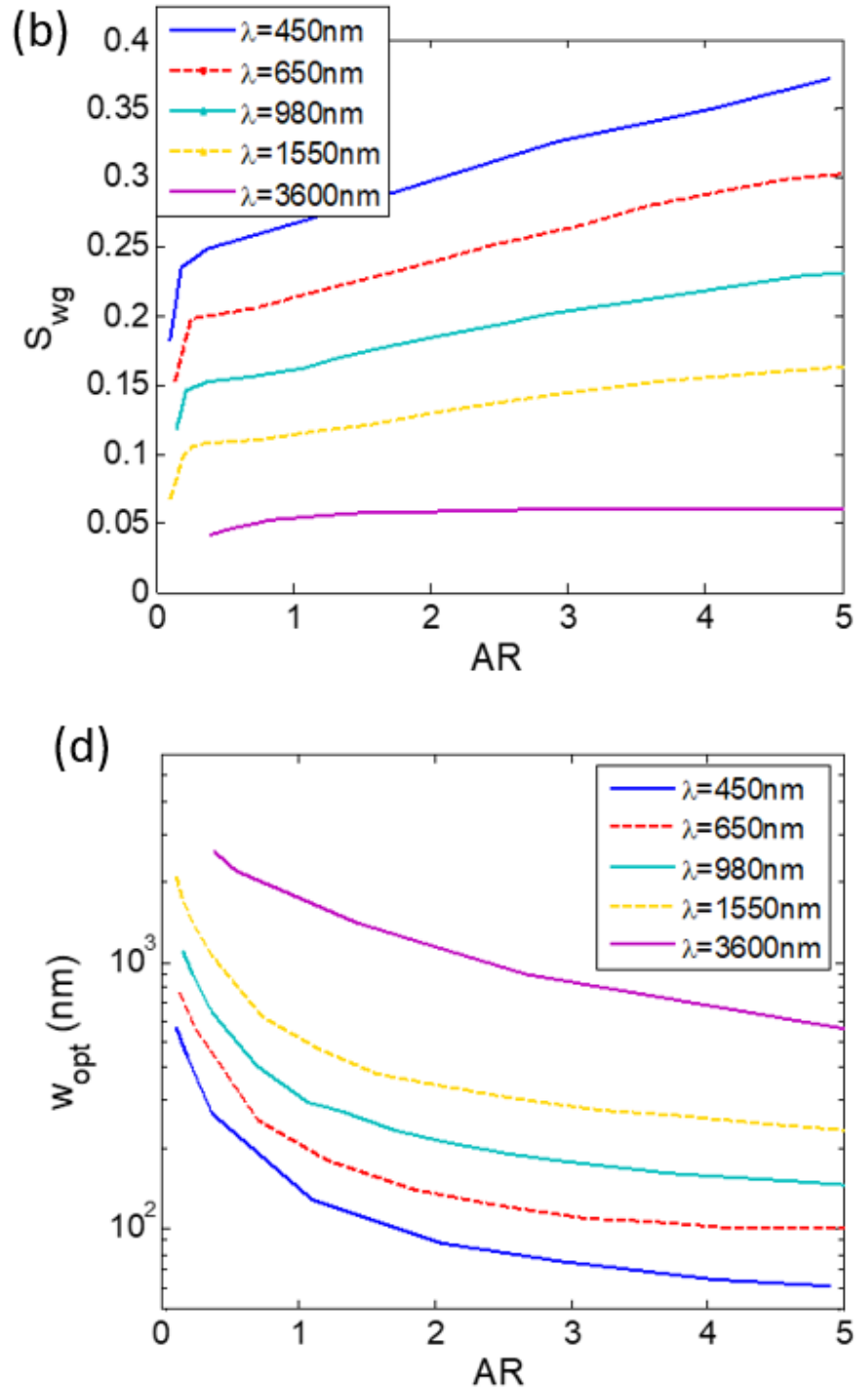

\section{Figure 3}

Strip waveguide sensitivity (Swg) versus waveguide aspect ratio (AR) for: (a) TE mode and (b) TM mode at different operating wavelengths. Optimum width (wopt) versus waveguide aspect ratio (AR) for: (c) TE mode and (d) TM mode at different operating wavelengths. 
(a)

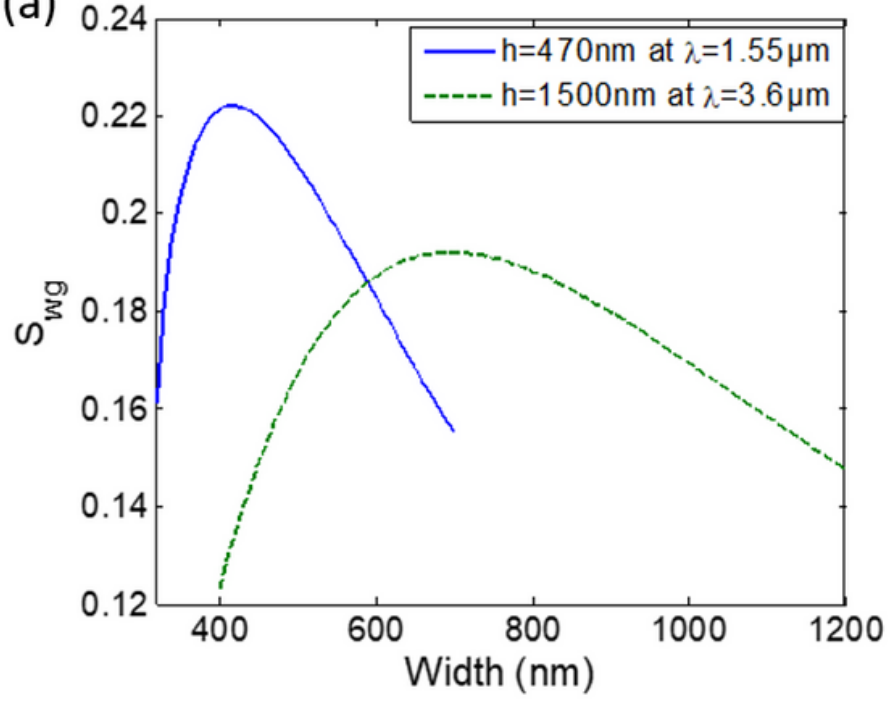

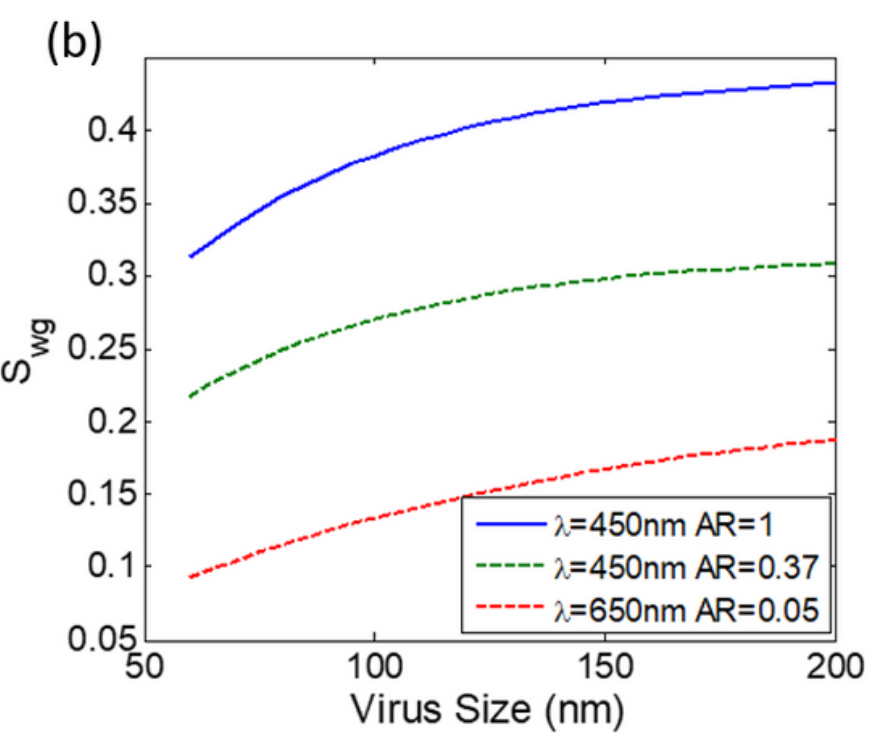

\section{Figure 4}

(a) Slot waveguide sensitivity Swg versus waveguide width the optimum waveguide thickness at $\lambda=1.55 \mu \mathrm{m}$ and $\lambda=3.6 \mu \mathrm{m}$ with wslot $=200 \mathrm{~nm}$. (b) Strip waveguide sensitivity Swg of TE mode (solid) and TM mode (dashed) versus virus size for different optimized designs.
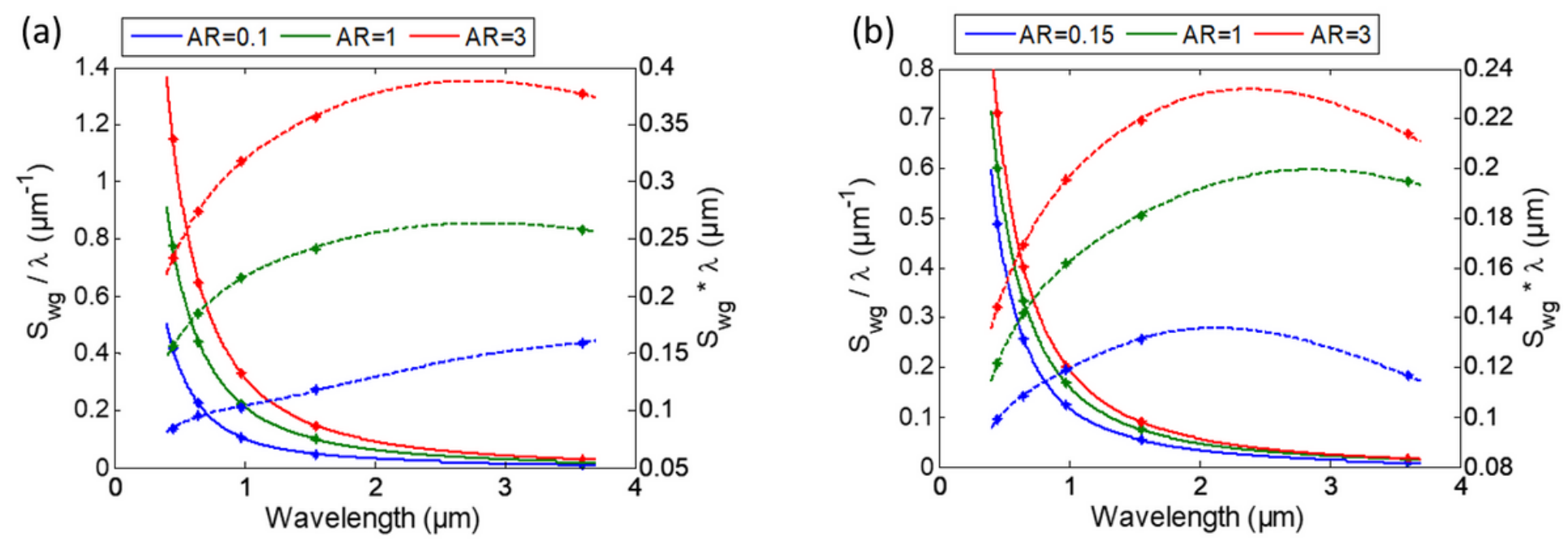

Figure 5

Fitted Swg $/ \lambda$ (solid line) and Swg $\times \lambda$ (dashed line) of strip waveguide versus wavelength at different AR with $\left.{ }^{*}\right)$ representing the simulated results for: (a) TE mode and (b) TM mode. 
(a)

$E_{\text {in }}$

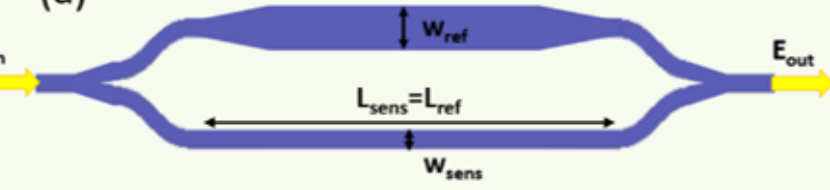

(b)

$E_{\text {in }}$

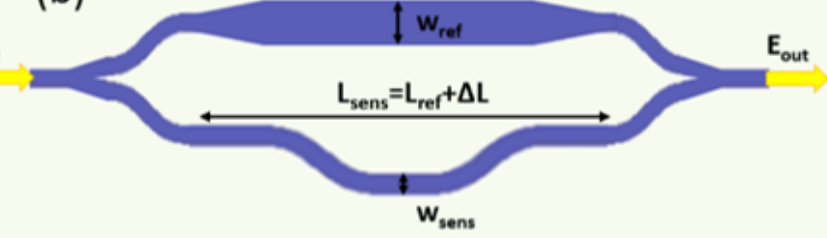

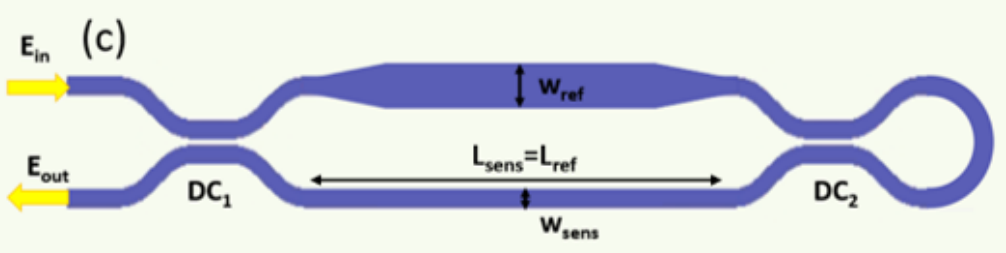

Figure 6

Schematic of MZI sensor configurations: (a) s-MZI, (b) a-MZI and (c) LT-MZI.

(a)

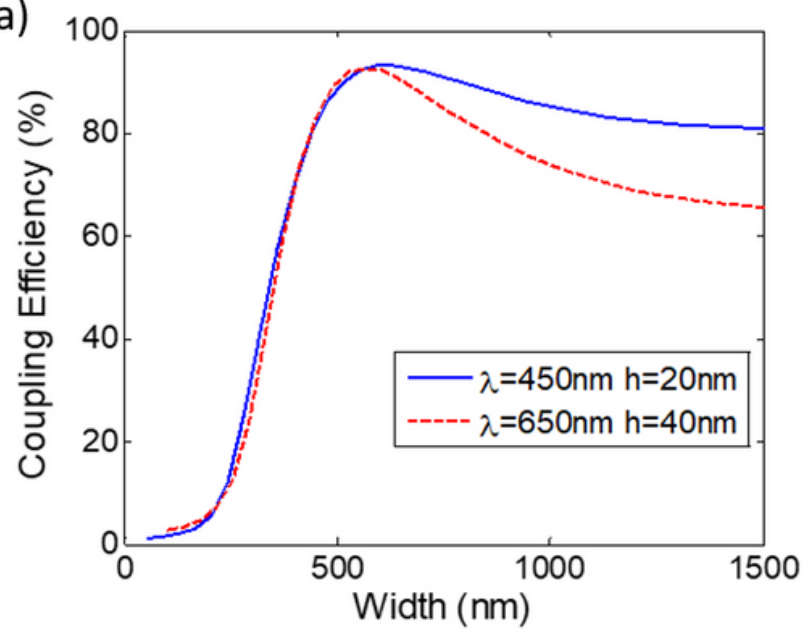

(b)

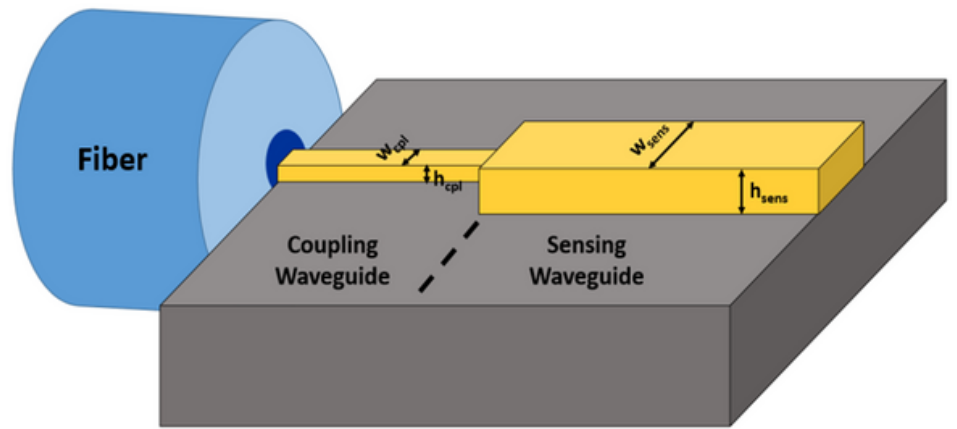

Figure 7

(a) Fiber to strip waveguide coupling efficiency versus waveguide width at $\lambda=450 \mathrm{~nm}$ and $\lambda=650 \mathrm{~nm}$ with $\mathrm{h}=20 \mathrm{~nm}$ and $\mathrm{h}=40 \mathrm{~nm}$, respectively. (b) Schematic of two-step fiber edge coupling. 